\title{
Broadband polarimetry of novae in outburst ${ }^{\star}$
}

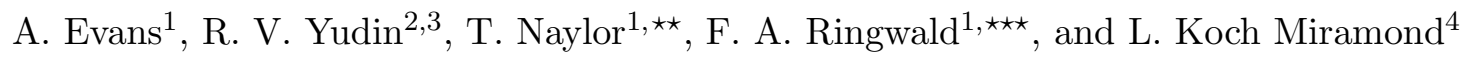 \\ 1 Department of Physics, Keele University, Keele, Staffordshire ST5 5BG, UK \\ 2 Central Astronomical Observatory of the Russian Academy of Sciences at Pulkovo, 196140 Saint-Petersburg, \\ Russia \\ 3 Isaac Newton Institute of Chile, St.-Petersburg Branch \\ 4 Service d'Astrophysique, CEA/DAPNIA, CE Saclay, 91191 Gif-sur-Yvette Cedex, France
}

Received 17 December 2001 / Accepted 7 January 2002

\begin{abstract}
We present broadband optical polarimetry of the classical novae V705 Cas, V4362 Sgr, V2313 Oph and BY Cir in outburst. The data indicate that, in all cases, there is an intrinsic component of polarization and in some cases the variability is very rapid, on a time-scale $\sim 1$ day. In the case of V705 Cas, we suggest that the origin of the intrinsic component may lie in the clumpiness and/or inhomogeneities of the ejecta, while electron scattering is the most likely cause in V2313 Oph. The wavelength-dependence of polarization in the case of V4362 Sgr suggests scattering by small dust grains, while polarization in resonance lines is the most probable cause of the observed polarization in BY Cir.
\end{abstract}

Key words. polarization - stars: circumstellar matter - stars: novae, cataclysmic variables

\section{Introduction}

Spectroscopy of classical novae during eruption (e.g. Hutchings 1972; Boyarchuk \& Gershberg 1977), direct imaging of nova shells when they are resolved (e.g. Dürbeck 1988; Paresce 1993; Paresce et al. 1995; Slavin et al. 1995), and theoretical considerations of nova winds (Livio et al. 1990; Porter et al. 1998) all demonstrate that material in a nova eruption is ejected anisotropically. The emission from classical novae during eruption might therefore be expected to be polarized, as a result of electron scattering, or of Mie scattering by dust grains condensing in the ejecta. Detailed calculations along these lines are given by Osman (1995). Polarimetry of novae during eruption can therefore carry information about the distribution of material in the ejecta which can complement spectroscopic information and, in the case of dusty

Send offprint requests to: A. Evans,

e-mail: ae@astro.keele.ac.uk

* Table 2 is also available in electronic form at the CDS via anonymous ftp to cdsarc.u-strasbg.fr (130.79.128.5) or via

http://cdsweb.u-strasbg.fr/cgi-bin/qcat?J/A+A/384/504

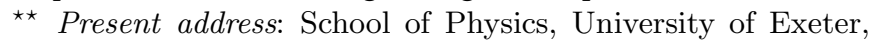
Stocker Road, Exeter EX4 4QL, UK.

$\star \star \star$ Present address: Department of Physics, California State University, Fresno, 2345 E. San Ramon Ave., MS MH37 Fresno, CA 93740-8031, USA. novae, provide information about the size and nature of grains. Even where the nova is not intrinsically polarized, polarization data, combined with data for field stars, can provide useful constraints on the interstellar reddening of novae, providing an independent check on reddening values determined by optical or ultraviolet methods.

Despite the potential information content of polarimetry, polarimetric observations of classical novae in outburst remain relatively rare, certainly in comparison to optical, infrared and ultraviolet studies (see Kikuchi et al. 1988; Bjorkman et al. 1994; Johnson et al. 1997; Ikeda et al. 2000, and references therein, for a comprehensive reference list).

We describe here broadband polarimetric observations of novae during eruption, obtained at the Pic du Midi (PdM) and South African Astronomical Observatories (SAAO).

\section{Observations and analysis}

\subsection{The novae}

The novae observed are listed in Table 1; times of maximum light (usually of discovery) are also listed. The observations cover the immediate post-outburst phase (BY Cir) to $\gtrsim 200$ days after outburst (V705 Cas). A number of field stars were also observed; finding charts and identifications are given in Fig. 1. Light curves, marked with the 

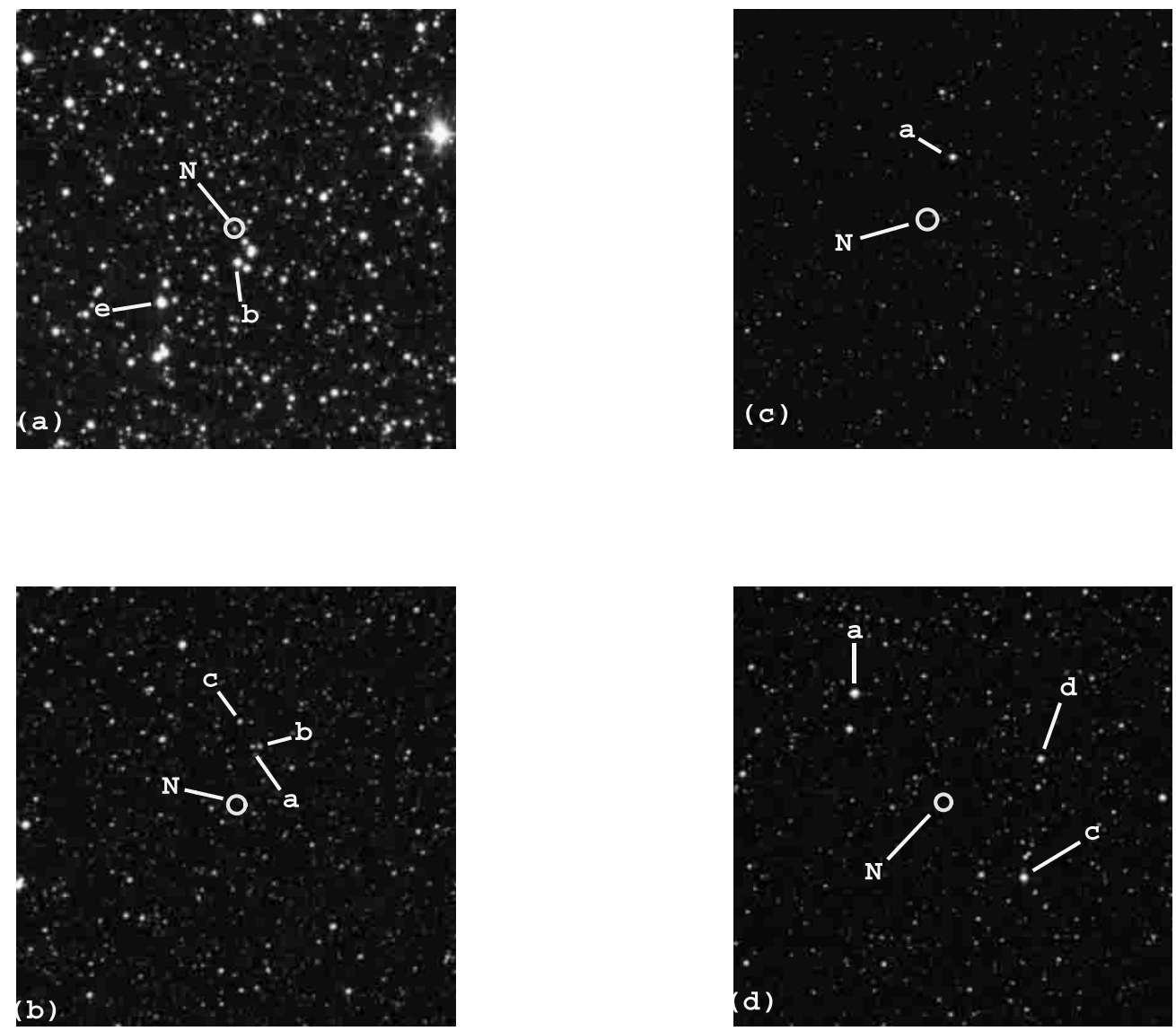

Fig. 1. Finding charts for novae (o) and field stars (a, b, etc.). a) V705 Cas; b) V4362 Sgr; c) V2313 Oph; d) BY Cir. Scale is $10^{\prime} \times 10^{\prime}$; north up, east left.

Table 1. Novae observed.

\begin{tabular}{llc}
\hline \multicolumn{1}{c}{ Object } & & $\begin{array}{c}\text { JD of } \\
\text { Maximum }\end{array}$ \\
\hline Nova Cas 1993 & V705 Cas & 49335 \\
Nova Sgr 1994 \#2 & V4362 Sgr & 49492 \\
Nova Oph 1994 & V2313 Oph & 49504 \\
Nova Cir 1995 & BY Cir & 49745 \\
\hline
\end{tabular}

times of the polarimetric observations reported here, are given in Fig. 2.

\subsection{Pic du Midi}

The observations at the PdM were carried out with the Sterenn polarimeter (Le Borgne 1987) at the Cassegrain focus of the $2 \mathrm{~m}$ Bernard Lyot telescope during the period 1994 July 2-July 15. Details of the modes of operation of the Sterren polarimeter may be found in Le Borgne (1987), in Koch Miramond \& Naylor (1995) and Naylor et al. (1996). The observations were carried out through a $B$ filter; the instrumental polarization is $0.062 \pm 0.006 \%$ in the $B$-band (see Koch Miramond \& Naylor 1995). Polarimetric standards were taken from the list of Hsu \& Breger (1982).

\section{3. $S A A O$}

Observations at SAAO were carried out during 1994 August 2-15 and 1995 January 23-February 6. The University of Cape Town (UCT) photometer-polarimeter module (Cropper 1985) was used on the SAAO $0.75 \mathrm{~m}$ telescope, although the observations for the period 1994 August 9-15 were carried out on the SAAO $1 \mathrm{~m}$ telescope. The instrumental linear polarization at $U B V$ has been measured by Clarke et al. (1998), who find it to be $0.71 \%(U), 0.28 \%(B), 0.09 \%(V)$. These values are somewhat higher than those reported by Cropper (1985, also D. Buckley, private communication) and may reflect longterm changes in the SAAO system. We have removed the instrumental polarization from our data using the instrumental polarization given in Clarke et al. (1998).

The majority of the observations were taken through $U B V R_{\mathrm{c}} I_{\mathrm{c}}$ filters, although a small number were taken through a clear $(C)$ filter. Polarimetric standards were again taken from Hsu \& Breger (1982). Each polarimetric observation with the UCT polarimeter also provides an estimate of the corresponding magnitude.

\subsection{Data analysis}

The data were reduced using standard packages provided by the observatories and the data are listed in Table 2 , in 

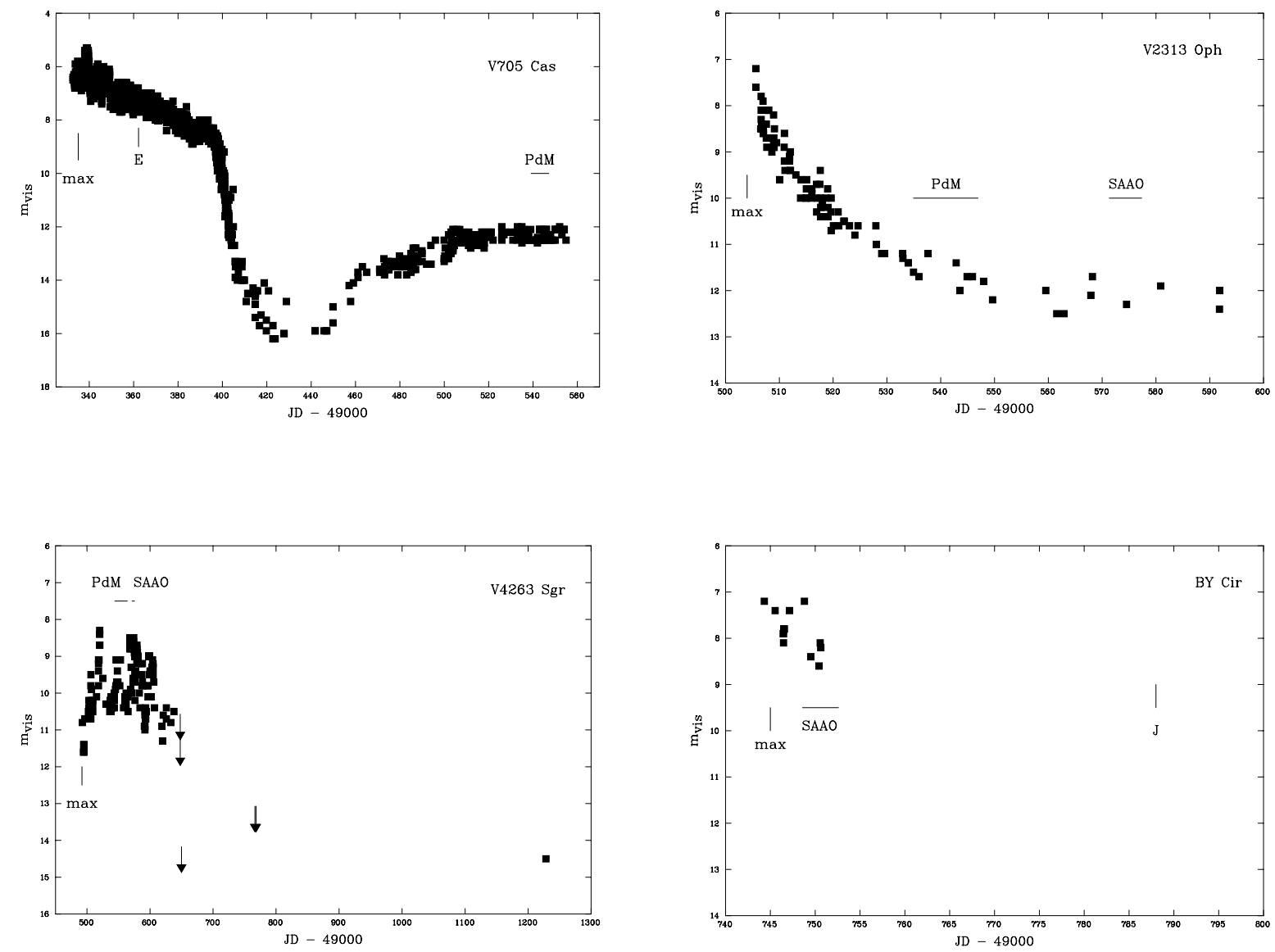

Fig. 2. Visual light curves for the novae. Times of the observations at PdM and SAAO are indicated. For BY Cir and V705 Cas the times of the observations by El'kin (1995; E) and Johnson et al. (1997; J) are also indicated.

which $t$ is the time (in days) since maximum light as given in Table 1. As times of maximum are usually uncertain to \pm 1 day, the value of $t$ is given only to the nearest 0.5 day. The Julian days are the time of mid-observation. The UCT magnitudes are also listed in Table 2.

The values of the normalized Stokes parameters (NSP) $q=Q / I$ and $u=U / I$ in Table 2 were corrected for instrumental polarization, noise bias etc. using the formalism of Clarke \& Stewart (1986). Further details of the data analysis may be found in Yudin \& Evans (1998) and Clarke et al. (1998). We tested for the presence of polarization using the $z$-statistic described in Clarke \& Stewart (1986); where polarization was detected at the $99 \%(\sim 3 \sigma)$ confidence level the values of the degree of polarization $P$ and equatorial position angle $\theta$ are given in Table 2 . The NSP (corrected for instrumental polarization) were also used to test for variability, using the Welch test (see Clarke \& Stewart 1986).

\section{Discussion}

\subsection{V705 Cas (1993)}

Nova Cas (V705 Cas) is a well-observed nova which was discovered before visual maximum. The time $t_{2}$ for the visual light curve to fall by two magnitudes from visual maximum, which is an indication of the energetics of the outburst (e.g. Warner 1995), was $\simeq 45$ days, placing it in the "moderately fast" speed class (see Fig. 2). Some 60 days after outburst, it went into deep minimum as carbon and silicate dust condensed in the ejecta (Shore et al. 1994). The observations described here cover the period around 200 days after maximum, after the nova had recovered from deep minimum but while it was still displaying strong dust emission from both carbon and silicate grains (Gehrz et al. 1995; Evans et al. 1997; Mason et al. 1998).

A polarimetric observation of V705 Cas in the $B$-band on day 26 - well before dust condensation - was reported by El'kin (1995), who found $P=1.25 \%$ at $\theta=69^{\circ}$ $(u=0.836 \pm 0.031 \%, q=-0.929 \pm 0.020 \%)$ in the $B$-band. These values are in broad agreement with those reported here. El'kin also presented polarimetric data on a number of field stars, and used these data to deduce a distance of $940 \mathrm{pc}$ for this nova. He also concluded that the bulk of the polarization at 26 days after outburst was interstellar in origin. Other authors have determined a larger distance to the nova, mostly on the basis of the well-known maximum magnitude rate-of-decline relationship (see e.g. Warner 1995 for a review) and reddening 
Table 2. Polarimetric data for novae.

\begin{tabular}{|c|c|c|c|c|c|c|c|c|c|}
\hline Object & JD & $t(\mathrm{~d})$ & Site & Filter & Mag & $q(\%)$ & $u(\%)$ & $P(\%)$ & $\theta\left(^{\circ}\right)$ \\
\hline \multirow[t]{13}{*}{ V4362 Sgr } & 49543.99 & 51.0 & PdM & $B$ & \multirow{13}{*}{9.46} & $0.83 \pm 0.06$ & $-0.28 \pm 0.07$ & $0.87 \pm 0.06$ & $170.7 \pm 2.1$ \\
\hline & 49544.96 & 52.0 & PdM & $B$ & & $-0.05 \pm 0.04$ & $-0.69 \pm 0.05$ & $0.69 \pm 0.04$ & $133.1 \pm 1.8$ \\
\hline & 49546.01 & 54.0 & $\mathrm{PdM}$ & $B$ & & $-0.22 \pm 0.02$ & $-1.15 \pm 0.03$ & $1.17 \pm 0.03$ & $129.6 \pm 0.7$ \\
\hline & 49563.96 & 71.0 & PdM & $B$ & & $0.54 \pm 0.08$ & $-0.17 \pm 0.07$ & $0.57 \pm 0.08$ & $171.1 \pm 3.8$ \\
\hline & 49571.46 & 79.5 & SAAO & $B$ & & $-0.62 \pm 0.30$ & $-1.04 \pm 0.30$ & $1.21 \pm 0.30$ & $119.6 \pm 8.6$ \\
\hline & 49571.46 & 79.5 & SAAO & $V$ & & $-0.24 \pm 0.22$ & $-0.72 \pm 0.22$ & $0.76 \pm 0.22$ & $125.6 \pm 6.3$ \\
\hline & 49571.46 & 79.5 & SAAO & $R_{\mathrm{c}}$ & & $0.05 \pm 0.27$ & $-0.31 \pm 0.27$ & $0.27 \pm 0.27$ & $140 \pm 29$ \\
\hline & 49571.46 & 79.5 & SAAO & $I_{\mathrm{c}}$ & & $0.43 \pm 0.27$ & $-0.18 \pm 0.27$ & $0.27 \pm 0.27$ & $169 \pm 29$ \\
\hline & 49575.42 & 83.5 & SAAO & $U$ & & $0.95 \pm 0.47$ & $0.41 \pm 0.46$ & $1.04 \pm 0.47$ & $11.8 \pm 12.9$ \\
\hline & 49575.42 & 83.5 & SAAO & $B$ & & $0.34 \pm 0.23$ & $0.48 \pm 0.23$ & $0.59 \pm 0.23$ & $27.6 \pm 11.2$ \\
\hline & 49575.42 & 83.5 & SAAO & $V$ & & $0.35 \pm 0.14$ & $-0.17 \pm 0.14$ & $0.39 \pm 0.14$ & $167.2 \pm 10.3$ \\
\hline & 49575.42 & 83.5 & SAAO & $R_{\mathrm{c}}$ & & $0.57 \pm 0.12$ & $-0.19 \pm 0.12$ & $0.60 \pm 0.12$ & $170.8 \pm 5.8$ \\
\hline & 49575.42 & 83.5 & SAAO & $I_{\mathrm{c}}$ & & $0.57 \pm 0.09$ & $-0.37 \pm 0.09$ & $0.68 \pm 0.09$ & $163.4 \pm 3.8$ \\
\hline Star "a" & 49544.97 & - & PdM & $B$ & & $-0.27 \pm 0.05$ & $-0.17 \pm 0.04$ & $0.32 \pm 0.05$ & $106.0 \pm 0.4$ \\
\hline Star "b" & 49546.05 & - & $\mathrm{PdM}$ & $B$ & & $0.64 \pm 0.08$ & $-0.03 \pm 0.06$ & $0.64 \pm 0.07$ & $178.8 \pm 3.0$ \\
\hline \multirow[t]{5}{*}{ Star "c" } & 49546.06 & - & $\mathrm{PdM}$ & $B$ & & $0.35 \pm 0.05$ & $-1.17 \pm 0.04$ & $1.22 \pm 0.04$ & $143.4 \pm 1.0$ \\
\hline & 49575.43 & - & SAAO & $B$ & & $0.72 \pm 0.21$ & $-0.63 \pm 0.21$ & $0.96 \pm 0.21$ & $159.4 \pm 6.3$ \\
\hline & 49575.43 & - & SAAO & $V$ & & $0.49 \pm 0.12$ & $-1.15 \pm 0.11$ & $1.25 \pm 0.11$ & $146.5 \pm 2.5$ \\
\hline & 49575.44 & - & SAAO & $R_{\mathrm{c}}$ & & $0.57 \pm 0.10$ & $-1.44 \pm 0.10$ & $1.55 \pm 0.10$ & $145.7 \pm 1.8$ \\
\hline & 49575.44 & - & SAAO & $I_{\mathrm{c}}$ & & $0.39 \pm 0.08$ & $-1.19 \pm 0.08$ & $1.25 \pm 0.08$ & $144.0 \pm 1.8$ \\
\hline \multirow[t]{9}{*}{ V2313 Oph } & 49534.93 & 31.0 & $\mathrm{PdM}$ & $B$ & \multirow{11}{*}{12.06} & $-0.31 \pm 0.10$ & $0.78 \pm 0.09$ & $0.84 \pm 0.10$ & $55.7 \pm 3.2$ \\
\hline & 49534.93 & 31.0 & PdM & $B$ & & $-0.31 \pm 0.09$ & $0.80 \pm 0.08$ & $0.86 \pm 0.09$ & $55.6 \pm 2.9$ \\
\hline & 49542.97 & 39.0 & PdM & $B$ & & $-0.77 \pm 0.10$ & $0.70 \pm 0.14$ & $1.04 \pm 0.10$ & $69.0 \pm 2.8$ \\
\hline & 49545.99 & 42.0 & $\mathrm{PdM}$ & $B$ & & $-0.50 \pm 0.08$ & $0.78 \pm 0.08$ & $0.92 \pm 0.08$ & $61.3 \pm 2.3$ \\
\hline & 49546.91 & 43.0 & $\mathrm{PdM}$ & $B$ & & $-0.70 \pm 0.12$ & $0.50 \pm 0.17$ & $0.86 \pm 0.15$ & $72.4 \pm 4.8$ \\
\hline & 49546.92 & 43.0 & PdM & $B$ & & $-0.47 \pm 0.10$ & $0.60 \pm 0.10$ & $0.76 \pm 0.10$ & $64.2 \pm 3.7$ \\
\hline & 49571.32 & 67.0 & SAAO & $V$ & & $-0.26 \pm 0.71$ & $1.46 \pm 0.71$ & $1.48 \pm 0.71$ & $50.1 \pm 13.7$ \\
\hline & 49577.34 & 73.0 & SAAO & $R_{\mathrm{c}}$ & & $-0.55 \pm 0.26$ & $1.49 \pm 0.26$ & $1.59 \pm 0.26$ & $55.1 \pm 4.7$ \\
\hline & 49577.35 & 73.0 & SAAO & $I_{\mathrm{c}}$ & & $-0.63 \pm 0.40$ & $1.83 \pm 0.40$ & $1.94 \pm 0.40$ & $54.5 \pm 5.9$ \\
\hline \multirow[t]{2}{*}{ Star "a" } & 49546.94 & - & PdM & $B$ & & $-0.54 \pm 0.09$ & $0.81 \pm 0.09$ & $0.97 \pm 0.09$ & $61.9 \pm 2.6$ \\
\hline & 49571.33 & - & SAAO & $R_{\mathrm{c}}$ & & $-0.38 \pm 0.35$ & $0.44 \pm 0.35$ & $0.58 \pm 0.35$ & $65.5 \pm 17.3$ \\
\hline \multirow[t]{6}{*}{ V705 Cas } & 49539.10 & 204.0 & $\mathrm{PdM}$ & $B$ & & $-0.91 \pm 0.24$ & $1.26 \pm 0.28$ & $1.54 \pm 0.26$ & $63.0 \pm 4.8$ \\
\hline & 49539.11 & 204.0 & $\mathrm{PdM}$ & $B$ & & $-0.85 \pm 0.23$ & $1.22 \pm 0.27$ & $1.49 \pm 0.25$ & $62.5 \pm 4.7$ \\
\hline & 49539.11 & 204.0 & $\mathrm{PdM}$ & $B$ & & $-0.56 \pm 0.36$ & $1.13 \pm 0.35$ & $1.26 \pm 0.35$ & $58.3 \pm 7.6$ \\
\hline & 49543.11 & 208.0 & $\mathrm{PdM}$ & $B$ & & $-0.90 \pm 0.25$ & $1.08 \pm 0.22$ & $1.41 \pm 0.23$ & $64.9 \pm 4.6$ \\
\hline & 49545.11 & 210.0 & $\mathrm{PdM}$ & $B$ & & $-0.49 \pm 0.15$ & $0.91 \pm 0.15$ & $1.03 \pm 0.15$ & $59.1 \pm 3.9$ \\
\hline & 49547.08 & 212.0 & $\mathrm{PdM}$ & $B$ & & $-0.68 \pm 0.11$ & $1.02 \pm 0.10$ & $1.22 \pm 0.10$ & $61.8 \pm 2.4$ \\
\hline \multirow[t]{2}{*}{ Star "b" } & 49547.08 & - & PdM & $B$ & & $-1.03 \pm 0.47$ & $1.56 \pm 0.43$ & $1.87 \pm 0.45$ & $61.8 \pm 6.7$ \\
\hline & 49545.12 & - & $\mathrm{PdM}$ & $B$ & & $-0.64 \pm 0.45$ & $1.52 \pm 0.48$ & $1.65 \pm 0.56$ & $56.5 \pm 9.2$ \\
\hline \multirow[t]{2}{*}{ Star "e" } & 49547.13 & - & PdM & $B$ & & $-0.13 \pm 0.09$ & $1.22 \pm 0.15$ & $1.23 \pm 0.13$ & $48.0 \pm 2.9$ \\
\hline & 49545.13 & - & $\mathrm{PdM}$ & $B$ & & $-0.23 \pm 0.13$ & $1.31 \pm 0.10$ & $1.33 \pm 0.11$ & $50.0 \pm 2.4$ \\
\hline
\end{tabular}

determined from (for example) spectral line ratios. Thus Arkhipova et al. (2000) determined a distance of $1.17 \mathrm{kpc}$, while Lynch et al. (1997) find $1.25 \pm 0.29 \mathrm{kpc}$; Hric et al. (1998) deduced $1.67 \pm 0.34 \mathrm{kpc}$, although Eyres et al. (2000) find $570 \pm 40 \mathrm{pc}$ from the expansion of the radio remnant. Assuming a distance in the range 1000-1500 pc we estimated an interstellar component of polarization for the nova using the catalogue of Heiles (2000) and our measurements of field stars as $p_{\text {is }}=1.40 \pm 0.15 \%$ and $\theta_{\text {is }}=58^{\circ} \pm 2^{\circ}$. Assuming these values we calculated an intrinsic component of polarization from the data of El'kin $\left(p_{\text {int }}=0.52 \%\right.$ at $\left.\theta_{\text {int }}=117^{\circ}\right)$ and from our average data $\left(p_{\text {int }}=0.19 \%\right.$ at $\left.\theta_{\text {int }}=114^{\circ}\right)$. In contrast to the conclusion of El'kin an intrinsic polarization for the nova is clearly present on day 26 , at the $99 \%$ confidence level.

Within the errors no statistically significant changes in either of the Stokes parameters were detected during the 9 day period of our observations, allowing us to calculate 
Table 2. continued.

\begin{tabular}{|c|c|c|c|c|c|c|c|c|c|}
\hline Object & JD & $t(\mathrm{~d})$ & Site & Filter & Mag & $q(\%)$ & $u(\%)$ & $P(\%)$ & $\theta\left(^{\circ}\right)$ \\
\hline \multirow[t]{14}{*}{ BY Cir } & 49748.59 & 3.5 & SAAO & $\bar{V}$ & 9.19 & $-0.62 \pm 0.11$ & $4.16 \pm 0.11$ & $4.21 \pm 0.11$ & $49.2 \pm 0.7$ \\
\hline & 49748.60 & 3.5 & SAAO & $U$ & & $-1.07 \pm 0.29$ & $4.35 \pm 0.29$ & $4.48 \pm 0.29$ & $51.9 \pm 1.8$ \\
\hline & 49748.60 & 3.5 & SAAO & $B$ & & $-0.04 \pm 0.40$ & $3.66 \pm 0.40$ & $3.66 \pm 0.40$ & $45.3 \pm 3.1$ \\
\hline & 49748.60 & 3.5 & SAAO & $V$ & 9.19 & $-0.49 \pm 0.11$ & $3.98 \pm 0.11$ & $4.01 \pm 0.11$ & $48.5 \pm 0.8$ \\
\hline & 49749.58 & 4.5 & SAAO & $U$ & & $-0.88 \pm 0.08$ & $3.82 \pm 0.08$ & $3.92 \pm 0.08$ & $51.5 \pm 0.6$ \\
\hline & 49749.59 & 4.5 & SAAO & $B$ & & $-0.29 \pm 0.11$ & $3.90 \pm 0.11$ & $3.91 \pm 0.11$ & $47.1 \pm 0.8$ \\
\hline & 49749.59 & 4.5 & SAAO & $V$ & 9.02 & $-0.47 \pm 0.11$ & $3.74 \pm 0.11$ & $3.77 \pm 0.11$ & $48.6 \pm 0.8$ \\
\hline & 49751.59 & 6.5 & SAAO & $U$ & & $-1.49 \pm 0.32$ & $4.09 \pm 0.32$ & $4.35 \pm 0.32$ & $55.0 \pm 2.1$ \\
\hline & 49751.59 & 6.5 & SAAO & $B$ & & $-0.67 \pm 0.43$ & $4.23 \pm 0.43$ & $4.28 \pm 0.43$ & $49.5 \pm 2.9$ \\
\hline & 49751.59 & 6.5 & SAAO & $V$ & 9.20 & $-0.67 \pm 0.13$ & $3.96 \pm 0.13$ & $4.02 \pm 0.13$ & $49.8 \pm 0.9$ \\
\hline & 49751.58 & 6.5 & SAAO & C & & $-0.46 \pm 0.10$ & $3.94 \pm 0.10$ & $3.97 \pm 0.10$ & $48.3 \pm 0.7$ \\
\hline & 49752.58 & 7.5 & SAAO & $U$ & & $-1.09 \pm 0.32$ & $4.11 \pm 0.32$ & $4.25 \pm 0.32$ & $52.4 \pm 2.2$ \\
\hline & 49752.59 & 7.5 & SAAO & $B$ & & $-0.28 \pm 0.42$ & $4.28 \pm 0.42$ & $4.29 \pm 0.42$ & $46.9 \pm 2.8$ \\
\hline & 49752.59 & 7.5 & SAAO & $V$ & 9.42 & $-0.46 \pm 0.13$ & $3.68 \pm 0.13$ & $3.71 \pm 0.13$ & $48.6 \pm 1.0$ \\
\hline \multirow[t]{4}{*}{ Star "a" } & 49748.62 & - & SAAO & $V$ & & $-1.28 \pm 0.15$ & $3.34 \pm 0.15$ & $3.58 \pm 0.15$ & $55.5 \pm 1.2$ \\
\hline & 49749.60 & - & SAAO & $U$ & & $-0.25 \pm 0.23$ & $2.69 \pm 0.23$ & $2.70 \pm 0.23$ & $47.7 \pm 2.4$ \\
\hline & 49749.60 & - & SAAO & $B$ & & $-0.39 \pm 0.39$ & $3.21 \pm 0.39$ & $3.23 \pm 0.39$ & $48.2 \pm 3.5$ \\
\hline & 49749.60 & - & SAAO & $V$ & & $0.45 \pm 0.10$ & $2.90 \pm 0.11$ & $2.93 \pm 0.11$ & $40.6 \pm 1.1$ \\
\hline \multirow[t]{3}{*}{ Star "c" } & 49752.60 & - & SAAO & $U$ & & $-0.97 \pm 0.36$ & $4.15 \pm 0.36$ & $4.26 \pm 0.36$ & $51.6 \pm 2.4$ \\
\hline & 49752.60 & - & SAAO & $B$ & & $-0.03 \pm 0.54$ & $4.14 \pm 0.54$ & $4.14 \pm 0.54$ & $45.2 \pm 3.7$ \\
\hline & 49752.60 & - & SAAO & $V$ & & $-0.35 \pm 0.16$ & $3.56 \pm 0.16$ & $3.58 \pm 0.16$ & $47.8 \pm 1.3$ \\
\hline \multirow[t]{3}{*}{ Star "d" } & 49751.60 & - & SAAO & $U$ & & $-1.26 \pm 0.81$ & $0.73 \pm 0.81$ & $1.46 \pm 0.81$ & $75 \pm 16$ \\
\hline & 49751.60 & - & SAAO & $B$ & & $-1.41 \pm 0.77$ & $0.25 \pm 0.77$ & $1.43 \pm 0.77$ & $85 \pm 15$ \\
\hline & 49751.61 & - & SAAO & $V$ & & $-1.04 \pm 0.34$ & $1.24 \pm 0.34$ & $1.62 \pm 0.34$ & $65 \pm 6$ \\
\hline
\end{tabular}

a weighted average value. Although the average value of polarization $\left(\overline{p_{\mathrm{obs}}}=1.28 \pm 0.12 \%\right.$ and $\left.\overline{\theta_{\mathrm{obs}}}=61.8 \pm 2.5\right)$ is close to the interstellar value, the difference between them is significant at the $95 \%$ confidence level. Besides, our mean value of polarization differs from the value determined from El'kin's data, again at the $99 \%$ confidence level. All this indicates the presence of an intrinsic component of polarization.

We note that the intrinsic polarization decreased between day 26 and day 204-212. It might be argued that the intrinsic polarization of the nova was larger at the time of the El'kin observation than that reported here as an optically thick carbon dust shell formed around day 66 (Shore et al. 1994). However our data are not adequate to distinguish between scattering by nova dust grains or electron scattering as the origin of the intrinsic polarization. In any case, the presence of intrinsic polarization on day 26 points to nonspherical or clumpy ejecta very soon after outburst. Indeed Evans et al. (1996) argued that the ejecta must be clumpy at early stages of the eruption in order that the chemistry required for molecule and grain formation may proceed. For the period of our PdM observations the envelope became more uniform and/or more symmetric.

\subsection{V2313 Oph (1994)}

This was a fast nova, with a $t_{2}$ of approximately 6.5 days $\left(t_{3} \simeq 14\right.$ days); the light curve was smooth (see Fig. 2), characteristic of novae of this speed class. Optical spectra (Wagner et al. 1994; Benetti 1994; Stringfellow \& Filippenko 1994; van Dyk 1994; Albright 1994) showed complex H I and Fe II emission lines superposed on a flat continuum, identifying the nova as a member of the fast "Fe II" type in its early decline stage. Our PdM and SAAO observations cover the late decline.

Investigation of interstellar polarization in the vicinity of the nova leads to the value $p_{\text {is }} \approx 1 \%$ and $\theta_{\text {is }} \approx 60^{\circ}$. This value agrees in general with the data obtained at the PdM around days 31-43, indicating that a significant part of observed polarization has an interstellar origin. Note, however, that our precise observations at the PdM clearly show small-scale polarimetric variability. The variations are statistically significant at the $95 \%$ confidence level, with amplitudes in both Stokes parameters about $0.3 \%$. Changes in the $q-u$ plane are shown in Fig. 3b. This behaviour indicates the presence of intrinsic polarization (at the level of about $0.2-0.4 \%$ ) at a relatively early phase after outburst. The variations of the position angle of intrinsic polarization are very large (up to $60^{\circ}$ ), indicating the possible presence of inhomogeneities in the ejecta. Although the data obtained at the SAAO on days $67-73$ have very low accuracy, they correspond to a larger value of intrinsic polarization (up to $1 \%$ on average), with approximately the same position angle for that time. Thus we note a small polarimetric variability on a short (a few days) time-scale, and larger variations on a medium-term (a few weeks) time-scale. As fast novae are not, in general, dust producers, we conclude in this case that the intrinsic component of polarization is due to electron scattering. 

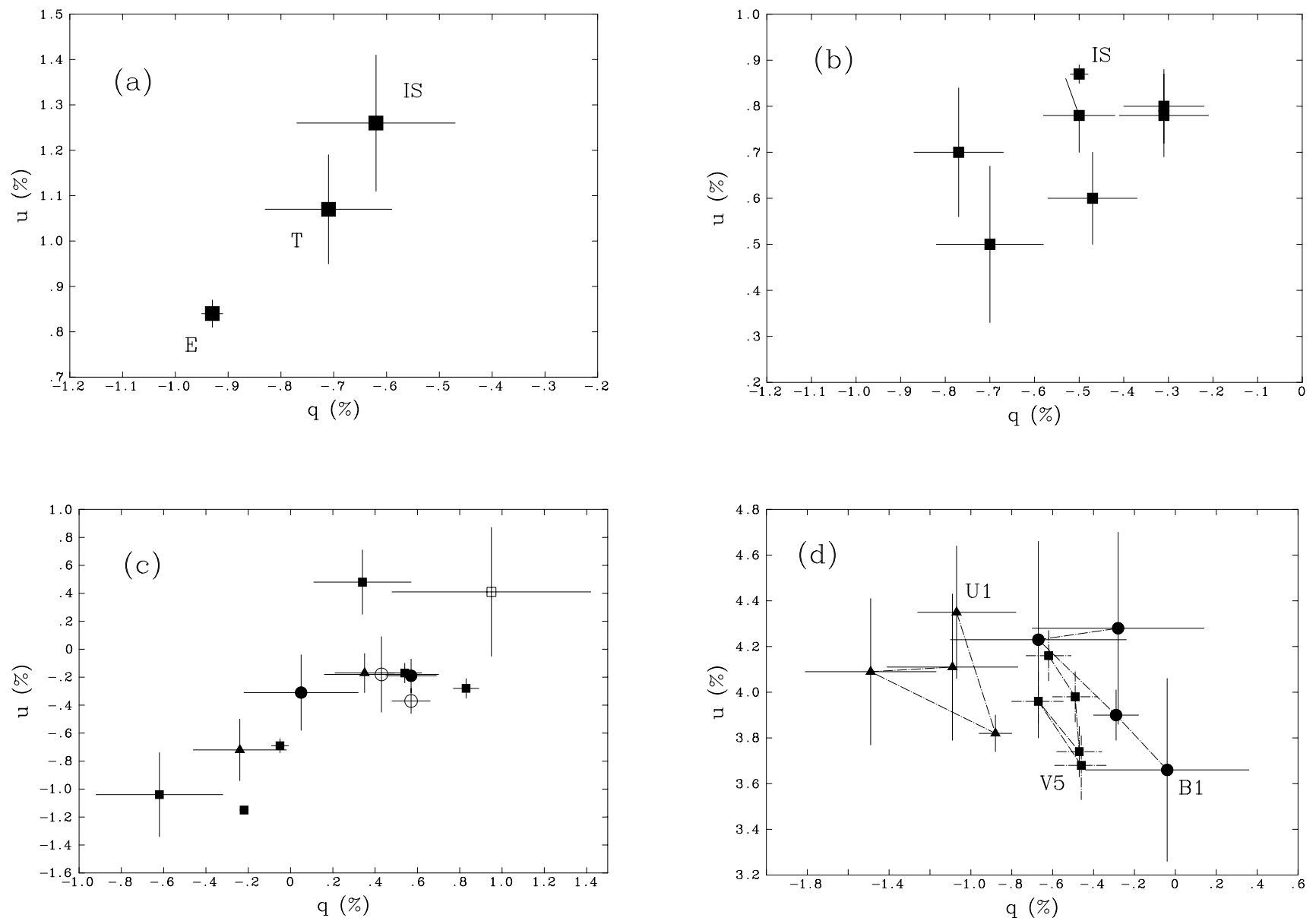

Fig. 3. a) Polarimetry for V705 Cas; "E" from El'kin (1995), "T" this paper. "IS" is the interstellar polarization. b) Polarimetric variations in the $q-u$ plane for V2313 Oph; "IS" is the interstellar polarization. c) Polarimetric variations in the $q-u$ plane for V4362 Sgr. Open squares $-U$, filled squares $-B$, filled triangles $-V$, filled circles $-R_{\mathrm{C}}$, open circles $-I_{\mathrm{C}}$. d) Polarimetric variations in the $q-u$ plane for BY Cir; triangles $-U$, circles $-B$, squares $-V$. Broken lines are included to guide the eye; "U1" is the first observation in the $U$ band etc. See text for details.

\section{3. $\operatorname{V4362~Sgr~(1994)~}$}

The light curve of this poorly-observed nova was erratic around maximum. Optical spectra obtained within a week of discovery (Austin et al. 1994) showed a nova with an early post-maximum "Fe II" class spectrum; these authors concluded that the widths of the emission lines suggested that the development of this nova would be moderately slow, consistent with the nature of the light curve. Although the visual light curve was only sparsely sampled, it may (like V705 Cas) have displayed a deep minimum, indicating the formation of an optically thick dust shell (see Fig. 2).

Our polarimetric observations of this nova cover the period from 51 days to 83 days after outburst. Unfortunately there is no information on the distance to this nova so we can not estimate an interstellar component of polarization. However the variations in the polarization parameters are unexpectedly large and rapid: the data obtained at the PdM over 4 nights, with high accuracy, show clear evidence of variability at the $99.9 \%$ confidence level. We are confident that the Sterenn instrument is extremely stable, as evidenced by the absence of variability, at the $\sim 0.01 \%$ level, in other targets observed during the same run (see Naylor et al. 1996). Changes are also evident in the data obtained a few days later at the SAAO, and so we have evidence of variable intrinsic polarization for V4362 Sgr at least from day 51. The variations in the $q-u$ plane are shown in Fig. 3c. These variations, with amplitudes of more than $1 \%$ for each of the Stokes parameters, are apparent on time-scales of a few days and indeed, there are significant changes on a time-scale $\sim 1$ day. We again deduce the presence of intrinsic polarization from the significant changes in the $q-u$ plane.

The variations of the NSP in the $B$-band are shown in Fig. 4a, together with the visual light curve over the same period. Unfortunately the sampling of the polarimetric variations is rather sparse and the visual magnitudes are mostly eye estimates with relatively large errors. Nevertheless Fig. 4a hints at correlated changes in the visual light and the NSP: significant variations in the position angle, up to $\approx 50^{\circ}$ (see also Table 2 ), occur as the nova brightens.

Multicolour data obtained at the SAAO with moderate accuracy allow us to investigate the 

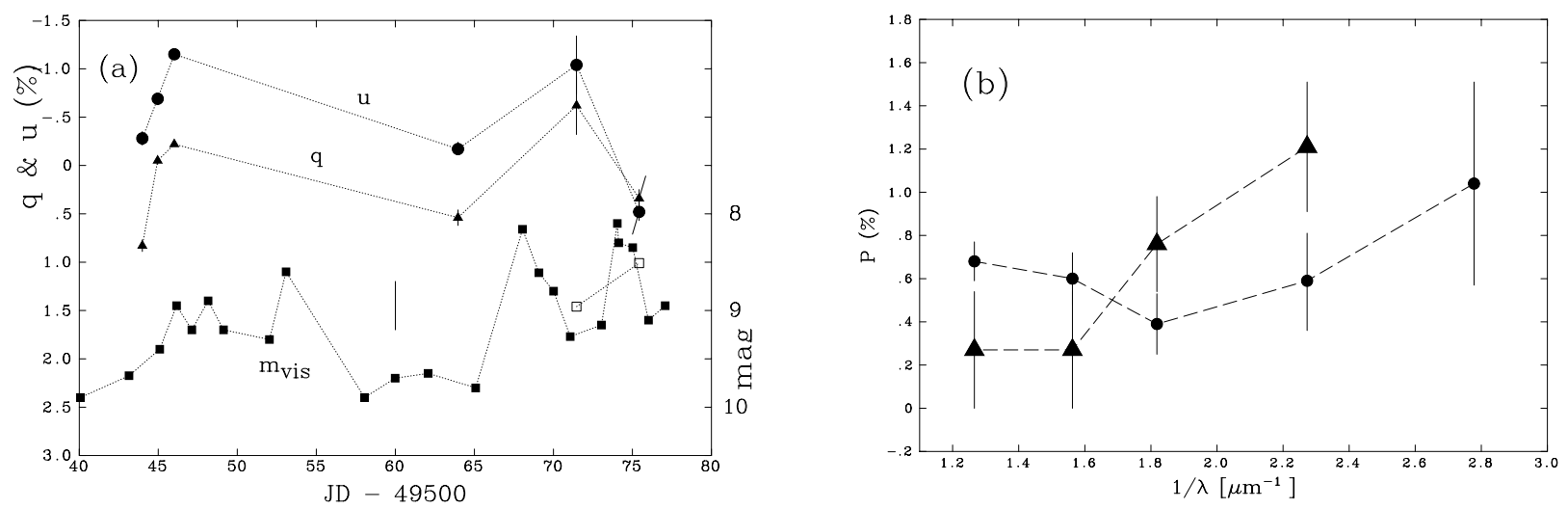

Fig. 4. a) Variations of $q$ and $u$ in the $B$-band, and the visual light curve of V4362 Sgr, for the period 49540-49580. The isolated "error bar" gives an indication of the range and uncertainty in the visual magnitudes; the open squares are photometry from SAAO. Error bars on polarimetry data are smaller than the plotted points if not included. Broken lines are included to guide the eye. b) Wavelength-dependence of polarization for V4362 Sgr on day 79.5 (triangles) and day 83.5 (circles). Broken lines are included to guide the eye between data taken on the same day.

wavelength-dependence of polarization for the nova (see Fig. 4b). This dependence does not correspond to electron scattering and the anomalous ratio $p(B) / p(V) \approx 1.5$ is consistent with scattering by small dust grains. The dates of the multicolour observations around 80 days after outburst correspond well to the time-scale for the formation of a carbon dust shell (e.g. $\sim 70$ days in the case of V705 Cas - see above), although the sparse evidence from the visual light curve (Fig. 2) suggests that large-scale dust condensation had not occurred at the time of our observations.

The rapid (on a time-scale $\sim 1$ day) and large polarimetric variations detected in the PdM data require significant changes in the circumstellar environment, or its illumination, on a very short time-scale. In particular the remarkable behaviour in the $q-u$ plane (see Fig. 3c), in which the data points are clearly concentrated along a straight line, indicate strong asymmetry of the ejected envelope. It is possible that the intrinsic polarization originates within narrow conical polar caps and/or in a flattened circular equatorial ring viewed nearly edge-on [see e.g. the recent discussions by Gill \& O'Brien (1999) and by Eyres et al. (2000) in the context of V705 Cas].

The wavelength-dependence of polarization (see above and Fig. 4) suggests scattering by small grains, which may be the precursors of the grains that eventually give rise to the deep minimum in the light curve (see Fig. 2). The correlations we see between the NSP and the visual light curve (see Fig. 4) may reflect the varying dominance of polarization in different regions of the circumstellar envelope. For example, if the increase in the visual flux is due to mass ejection into (say) the polar caps and the subsequent formation of small dust particles there, the scattering in this region would become dominant, leading to a rotation of the position angle of polarization. Further changes may arise if (for example) silicate and carbon condense in different regions of the ejecta (e.g. in rings and caps); scattering from one, then the other, dominates, leading to further changes of the position angle. Calculations along these lines have been carried out by Osman (1995), who finds that very rapid changes - particularly in position angle - should indeed occur as dust condenses.

\subsection{BY Cir (1995)}

Unfortunately this is another poorly-observed nova, with a poorly-determined light curve (see Fig. 2). An optical spectrum obtained close to maximum (Benetti \& Cappellaro 1995) showed a spectrum dominated by H I, Fe II and O II emission lines. Infrared photometry, obtained shortly after outburst, is reported by Zijlstra (1995).

Ultraviolet spectroscopy, obtained some 2 months after discovery, is reported by Greeley et al. (1995). They found strong emission in C II, C III, C IV, N III], N IV], N v, O III], O IV] and HeII. On the basis of the He II 1640/He II 1085 line ratio, they concluded that there was significant extinction at shorter wavelengths, corresponding to $E(B-V)=$ 0.11 . They further note that a similar value $[E(B-V)=$ $0.15]$ follows from the intrinsic and observed $(B-V)$ at maximum, from observations obtained within a day of those reported here (Gilmore 1995). Since any extinction at maximum is almost certainly interstellar, it is highly likely that the extinction reported by Greeley et al. (1995) was interstellar.

BY Cir is one of the few novae that have been observed spectropolarimetrically (Johnson et al. 1997). These authors, whose observations were carried out 44 days after discovery (i.e. some 40 days later than the observations reported here), carried out spectropolarimetry over the wavelength range $\sim 1500 \ldots 3000 \AA$ with the WUPPE polarimeter on ASTRO-2. They found that the intrinsic polarization in the continuum (after removing the interstellar contribution) was $P \simeq 0.4 \%$ at $\theta \simeq 17^{\circ}$, and that the polarization changes across the $\mathrm{Mg}$ II $\lambda 2800$ resonance line. The estimation of interstellar polarization used by these authors was $p_{\text {is }}=4.0 \%$ and $\theta_{\text {is }}=50.0^{\circ}$. Note that, according to the $p_{\text {is }}-D$ relation derived from the investigation 
Table 3. Intrinsic polarization for Nova BY Cir.

\begin{tabular}{ccccrrr}
\hline JD & \multicolumn{2}{c}{$U$} & \multicolumn{2}{c}{$B$} & \multicolumn{2}{c}{$V$} \\
& $P(\%)$ & $\theta\left(^{\circ}\right)$ & $P(\%)$ & $\theta\left(^{\circ}\right)$ & $P(\%)$ & $\theta\left(^{\circ}\right)$ \\
\hline 49748.59 & & & & & $0.23 \pm 0.11$ & $36 \pm 1$ \\
49748.60 & $1.54 \pm 0.29$ & $56 \pm 2$ & $0.62 \pm 0.40$ & $177 \pm 3$ & $0.20 \pm 0.11$ & $6 \pm 1$ \\
49749.59 & $0.97 \pm 0.08$ & $56 \pm 1$ & $0.41 \pm 0.11$ & $13 \pm 1$ & $0.30 \pm 0.11$ & $159 \pm 1$ \\
49751.59 & $1.53 \pm 0.32$ & $65 \pm 2$ & $0.51 \pm 0.43$ & $46 \pm 3$ & $0.03 \pm 0.13$ & $23 \pm 1$ \\
49752.59 & $1.32 \pm 0.32$ & $58 \pm 2$ & $0.69 \pm 0.42$ & $27 \pm 3$ & $0.35 \pm 0.13$ & $156 \pm 1$ \\
\hline
\end{tabular}

of field stars in the vicinity of BY Cir, this value of $p_{\text {is }}$ leads to a distance for the nova greater than $2-2.5 \mathrm{kpc}$.

Our observations of BY Cir were carried out within days of outburst. The polarization is high $(\sim 4 \%)$ at all wavelengths but the degree of polarization and the position angle are similar to those of the field stars, indicating that the polarization of BY Cir is largely interstellar in origin. However there seem to be changes in the $q-u$ plane (see Fig. 3d), at least in the $V$ band, which we regard as statistically significant at the $99 \%$ confidence level. Although five $q$ values in this band are indistinguishable within the errors, the $u$ parameter does show variation with an amplitude about $0.4 \%$. The errors in the $U B$ bands are too large to draw any conclusions about the variability.

Taking into account the value of interstellar polarization in the $V$ band, we estimated the values of $p_{\text {is }}$ in the $U B$ bands using the Serkowski law (see e.g. Whittet 1992), and subtracted them from our data using the component Stokes parameters. Thus we have calculated values of intrinsic polarization for BY Cir, which are given in Table 3.

As seen from Table 3, BY Cir shows a significant increase intrinsic polarization in the $U$ band, up to $1.5 \%$. The values of intrinsic polarization in the $U$ band calculated here agree well with the spectropolarimetric data of Johnson et al. (1997). As follows from their Fig. 4, the intrinsic polarization reaches a value $\simeq 1.4 \%$ around $\lambda \approx 3100 \AA$, whereas the continuum polarization does not exceed $0.4 \%$. Note that the unusual wavelength dependence of intrinsic polarization detected here is not typical for electron scattering. Therefore, the large polarization in the $U B$ bands close to maximum can not be attributed to a nonspherical circumstellar envelope, like that around classical Be stars (see e.g. Yudin 2001 and references therein). We conclude that the main contribution to the large intrinsic polarization in the $U B$ bands is due to the effect of polarized radiation of resonance lines (for the first report of such behaviour, see Johnson et al. 1997).

In the general interstellar medium we have that

$\frac{P\left(\lambda_{\max }\right)}{E(B-V)} \lesssim 0.09 \mathrm{mag}^{-1}$

where $P\left(\lambda_{\max }\right)$ is the maximum (with respect to wavelength) polarization and $E(B-V)$ is the reddening (e.g. Whittet 1992). We note that the polarization of BY Cir in the $C$ filter $\left(P_{\mathrm{c}}\right)$ on JD 49751.58 (see Table 2) was $3.97 \pm$ $0.10 \%$. $P_{\mathrm{c}}$ is usually about $80 \%$ of $P\left(\lambda_{\max }\right)$, (P. Barrett, private communication), which indicates that $P_{\max }$ for BY Cir was about $4.95 \%$. If the polarization of BY Cir was mostly interstellar, this implies that $E(B-V) \gtrsim 0.55$.

There is a clear discrepancy between the extinction as determined from the He II line ratio (Greeley et al. 1995) and the polarimetry, which is too large to be accounted for by uncertainties in the electron density and temperature assumed to determine the intrinsic He II line ratios. The discrepancy is exacerbated if (for example) the He II $\lambda 1640$ line were optically thick. An alternative explanation is that the ratio of total-to-selective extinction $R$ in the direction of BY Cir differs from the standard value ( $R=3.1$ ) assumed by Greeley et al. However if we take the suggested dependence of $R$ on Galactic longitude suggested by Whittet (1977), we find $R \simeq 3.2$ in the direction of BY Cir. The effect is to flatten the extinction law in the far ultraviolet (see Cardelli et al. 1989), which would make the discrepancy worse.

Although we do not have sufficient information on this nova to know whether or not it was a dust-producer our observations were obtained close to maximum, when we can be confident that no dust would have been produced. The polarimetry of BY Cir reported here, at $\sim 3-7$ days after discovery, is amongst the earliest with regard to nova development. The low continuum ( $V$-band) polarization close to maximum most likely indicates that the ejected material was close to being spherically symmetric at this time. This further suggests that the shaping of nova shells, as seen in optical images (e.g. Slavin et al. 1995), takes place well after eruption, consistent with the various models for this phenomenon (e.g. Livio et al. 1990; Porter et al. 1998).

\section{Concluding remarks}

We have presented broadband polarimetry of four classical novae (V705 Cas, V2313 Oph, V4362 Sgr and BY Cir) in eruption. There is evidence for intrinsic and variable polarization in all objects, and an anomaly in the reddening and wavelength dependence of polarization in the case of BY Cir. We conclude that intrinsic polarization for three of these novae is evident in the early stages after outburst (around days 30-70). In the case of V705 Cas, this is likely due to clumpiness and/or inhomogeneities in the ejecta, while in the case of V2313 Oph it is probably due to electron scattering. Scattering by small grains in a non-spherical or non-uniform shell is the most likely 
explanation for V4362 Sgr, in which we see very rapid changes in polarization. For BY Cir high intrinsic polarization was detected in the $U B$ bands $3-5$ days after outburst, indicating the possible influence of polarized resonance lines in this spectral region.

Unfortunately it is still the case that, despite their potential diagnostic value, polarimetric observations of novae in outburst remain relatively rare. A sustained polarimetric campaign, such as that carried out by Piirola \& Korhonen (1979) for V1668 Cyg, on a nova that is also well-observed at optical and infrared wavelengths is still required.

In particular, the possible correlation between the polarimetric and photometric variations in V4362 Sgr show that more detailed, co-ordinated polarimetric and photometric observations of future novae are required.

Acknowledgements. We thank Prof. V. G. El'kin for information about his polarimetric observations of V705 Cas. We acknowledge generous awards of time by the allocation panels of the PdM and SAAO, and the Particle Physics and Astronomy Research Council (PPARC) for the provision of travel funds. We also thank the staff at the respective observatories for support. FAR was supported by the PPARC, RVY by a Royal Society grant. This work made use of the SIMBAD database.

\section{References}

Albright, G. E. 1994, IAUC, 6002

Arkhipova, V. P., Burlak, M. A., \& Esipov, V. F. 2000, AstL, 26,372

Austin, S. J., Schwarz, G., \& Starrfield, S. 1994, IAUC, 5993

Benetti, S. 1994, IAUC, 6002

Bjorkman, K. S., Johansen, K. A., Nordsieck, K. H., Gallagher, J. S., \& Barger, A. J. 1994, ApJ, 425, 247

Boyarchuk, A. A., \& Gershberg, R. E. 1977, SAAJ, 21, 257

Cardelli, J. A., Clayton, C. C., \& Mathis, J. S. 1989, ApJ, 345, 245

Clarke, D., Smith, R. A., \& Yudin, R. V. 1998, A\&A, 336, 604

Clarke, D., \& Stewart, B. G. 1986, Vistas Astron., 29, 27

Cropper, M. 1990, MNRAS, 212, 709

Dürbeck, H. W. 1988, ESO Messenger

El'kin, V. G. 1995, Astr. Lett., 21, 670

Evans, A., Geballe, T. R., Rawlings, J. M. C., \& Scott, A. D. 1996, MNRAS, 282, 1049

Evans, A., Geballe, T. R., Rawlings, J. M. C., Eyres, S. P. S., \& Davies, J. K. 1997, MNRAS, 292, 192
Eyres, S. P. S., Bode, M. F., O'Brien, T. J., Watson, S. K., \& Davis, R. J. 2000, MNRAS, 318, 1086

Gehrz, R. D., Greenhouse, M. A., Hayward, T. L., et al. 1995, 448, L119

Gilmore, A. G. 1995, IAUC, 6130

Greeley, B. W., Blair, W. P., \& Long, K. S. 1995, BAAS, 27, 835

Heiles, C. 2000, AJ, 119, 923 (vizier.u-strasbg.fr/vis-bin/Cat?II/226)

Hric, L., Petrik, K., Urban, Z., \& Hanzel, D. 1998, A\&AS, 133, 211

Hsu, J., \& Breger, M. 1982, ApJ, 262, 732

Hutchings, J. S. 1972, MNRAS, 158, 177

Ikeda, Y., Kawabata, K. S., \& Akitaya, H. 2000, A\&A, 355, 256

Johnson, J. J., Anderson, C. M., Bjorkman, K. S., et al. 1997, AJ, 113, 2200

Kikuchi, S., Mikami, Y, \& Kondo, M. 1988, PASJ, 40, 491

Koch Miramond, L., \& Naylor, T. 1995, A\&A, 296, 390

Le Borgne, J. F. 1987, Ph.D. Thesis, Université de Toulouse

Livio, M., Shankar, A., Burkert, A., \& Truran, J. W. 1990, ApJ, 356, L250

Lynch, D. K., Russell, R. W., Kellogg, K. C., Mazuk, A. L., \& Hanner, M. S. 1997, AJ, 113, 1391

Mason, C. G., Gehrz, R. D., Woodward, C. E., et al. 1998, ApJ, 494, 783

Naylor, T., Koch-Miramond, L., Ringwald, F. A., \& Evans, A. 1996, MNRAS, 282, 873

Osman, S. M. 1995, Ph.D. Thesis, Keele University

Paresce, F. 1994, A\&A, 282, L13

Paresce, F., Livio, M., Hack, W., \& Korista, K. 1995, A\&A, 299, 823

Piirola, V., \& Korhonen, T. 1979, A\&A, 79, 254

Porter, J. M., O'Brien, T. J., \& Bode, M. F. 1998, MNRAS, 296, 943

Shore, S. N., Starrfield, S., Gonzalez-Riestra, R., Hauschildt, P. H., \& Sonneborn, G. 1994, Nature, 369, 539

Slavin, A. J., O'Brien, T. J., \& Dunlop, J. S. 1995, MNRAS, 276,353

Stringfellow, G. S., \& Filippenko, A. V. 1994, IAUC, 6002

van Dyk, S. D. 1994, IAUC, 6002

Wagner, R. M., Vandlandingham, K. M., \& King, N. 1994, IAUC, 6002

Warner, B. 1995, Cataclysmic Variable Stars, Chapter 5 (Cambridge University Press)

Whittet, D. C. B. 1977, MNRAS, 180, 29

Whittet, D. C. B. 1992, Dust in the Galactic Environment (IoP Publishing, Bristol, Philadelphia, New York)

Yudin, R. V., \& Evans, A. 1998, A\&AS, 131, 401

Yudin, R. V. 2001, A\&A, 368, 912

Zijlstra, A. 1995, IAUC, 6131 